\title{
1 Translocational unfolding in clostridial binary iota toxin
}

\section{2 complex}

3 Tomohiro Yamada ${ }^{1,7}$, Toru Yoshida ${ }^{1,2,7}$, Akihiro Kawamoto ${ }^{3,7}$, Kaoru Mitsuoka ${ }^{4}$, Kenji

4 Iwasaki $^{3,5}$, and Hideaki Tsuge ${ }^{1,2,6, *}$

5

61 Faculty of Life Sciences, Kyoto Sangyo University, Kamigamo-motoyama, Kita-ku,

7 Kyoto, Japan.

82 Institute for Protein Dynamics, Kyoto Sangyo University, Kamigamo-motoyama,

9 Kita-ku, Kyoto, Japan.

103 Institute for Protein Research, Osaka University, Suita, Osaka, 565-0871, Japan.

114 Research Center for Ultra-High Voltage Electron Microscopy, Osaka University,

12 Osaka, Japan

135 Life Science Center for Survival Dynamics, Tsukuba Advanced Research Alliance

14 (TARA), University of Tsukuba, Tsukuba, Japan

156 Center for Molecular Research in Infectious Diseases, Kyoto Sangyo University,

16 Kamigamo-motoyama, Kita-ku, Kyoto, Japan.

177 These authors contributed equally: Tomohiro Yamada, Toru Yoshida, Akihiro

18 Kawamoto

19

20 *Correspondence: tsuge@cc.kyoto-su.ac.jp 


\section{Abstract}

23 Protein translocation across the membrane is critical for microbial

24 pathogenesis and various cellular functions. Bacterial binary toxins such as

25 anthrax toxin are composed of enzyme components and a translocation channel,

26 which catalyses substrate unfolding and translocation. Here we report the

27 structures of the clostridial binary toxin (iota toxin) translocation channel Ib-pore

28 and its complex with ADP-ribosyltransferase Ia. The Ib-pore structure at atomic

29 resolution provides a similar structural framework as observed for the catalytic

$30 \phi$-clamp of the anthrax protective antigen pore. However, the Ia-bound Ib-pore

31 structure showed a unique binding mode of Ia: one Ia binds to the Ib-pore, and the

32 Ia N-terminal domain interacts with Ib via two other Ib-pore bottlenecks with

33 multiple weak interactions. Furthermore, Ib-binding induces Ia N-terminal $\alpha$-helix

34 tilting and partial unfolding, whereupon the unfolded $\mathrm{N}$-terminus continues to the

$35 \phi$-clamp gate. This study reveals the novel mechanism of $\mathbf{N}$-terminal unfolding,

36 which is crucial for protein translocation. 


\section{Introduction}

40 Clostridium perfringens iota toxin (iota), C. difficile toxin ( CDT), C. spiroforme toxin

41 (CST), and C. botulinum $\mathrm{C} 2$ toxin belong to the family of binary toxins. It consists of

42 the enzymatic 'A' component with actin-specific ADP-ribosyltransferase and the 'B'

43 component which binds to the host cell and functions as the translocation channel of

44 each enzymatic component (Ia,Ib; CDTa,CDTb; CSTa,CSTb; and C2I,C2II,

45 respectively). The ' $\mathrm{B}$ ' component precursor is first cleaved off by a cellular protease,

46 then binds to the target cell via a receptor, forms a soluble oligomer termed the

47 'prepore', and finally converts to a membrane-spanning pore in an acidified endosome.

48 The oligomer-receptor complex acts as a substrate docking platform that subsequently

49 translocates an enzymatic component into the cytosol from the acidified endosome.

50 Therein, the enzymatic component mono-ADP-ribosylates G-actin, inducing

51 cytoskeletal disarray and cell death. The enzymatic component consists of two domains:

52 the C-terminal domain with an actin-specific ADP-ribosyltransferase activity, and the

$53 \quad \mathrm{~N}$-terminal domain, considered as a binding domain to the membrane-spanning

54 translocation component. The clostridial binary (iota) toxin consists of Ia and $\mathrm{Ib}^{1-3}$.

55 However, although extensive structural and functional studies of Ia have been

56 conducted $^{4,5}$, little is known regarding the Ib translocation channel.

57 A similar binary toxin, anthrax toxin, constitutes a major virulence factor of Bacillus

58 anthracis, consisting of an enzymatic component (two enzymatic proteins, edema (EF)

59 and lethal (LF) factors) and a protein translocation channel (protective antigen (PA) $)^{6}$.

60 LF is a zinc-dependent protease that cleaves mitogen-activated protein kinase kinase;

61 the combination of PA and LF leads to the death of humans and animals. Cleavage of a 
62 PA precursor triggers heptamerization or octamerization (prepore), and the low $\mathrm{pH}$

63 endosomal environment causes the oligomer to insert into the membrane by forming a

64 transmembrane $\beta$-barrel (pore) ${ }^{7}$. PA heptameric prepore structure was revealed with and

65 without anthrax toxin receptor ${ }^{8,9}$. Furthermore, the LF-bound PA prepore complex

66 structure (PA dimer with one LF N-terminal domain $\left(\mathrm{LF}_{\mathrm{N}}\right)$ ) was revealed by

67 crystallography at $3.1 \AA$ resolution. The biological unit structure $\left(\mathrm{PA}_{8}\left(\mathrm{LF}_{\mathrm{N}}\right)_{4}\right)$ was also

68 deduced from the complex, revealing that the first N-terminal helix of LF binds on the

69 PA dimer interface surface ${ }^{10}$.

70 Although PA pore instability has hampered its structural analysis, Jiang et al.

71 clarified the PA heptameric pore structure by cryo-electron microscopy (cryo-EM) at

$72 \quad 2.9 \AA$ resolution $^{11}$, revealing the catalytic $\phi$-clamp and a long membrane-spanning

73 channel $^{12}$. The $\phi$-clamp is the narrowest passageway of the PA translocation channel, in

74 which the seven phenylalanine-427 residues converge within the lumen, generating a

75 radially symmetric solvent-exposed aromatic ring. Krantz et al. proposed that the

$76 \phi$-clamp serves a chaperone-like function, interacting with hydrophobic sequences

77 presented by the protein substrate as it unfolds during translocation ${ }^{12}$. Because

78 translocation is driven by a transmembrane proton gradient, a Brownian ratchet model

79 has been proposed, which depends on protonation and deprotonation of the

80 translocating polypeptide acidic residues ${ }^{12-15}$. Furthermore, an allosteric

81 helix-compression model regulated by the $\alpha$-clamp in PA was recently proposed ${ }^{16}$,

82 although it remains controversial whether this model is adequate.

83 To our knowledge, no high-resolution structure of the enzyme-bound translocation

84 channel including the LF-bound PA-pore is available (although the PA heptamer pore 
85 structure with three $\mathrm{LF}_{\mathrm{N}}$ was reported at $17 \AA$ resolution ${ }^{17}$ ), and pore structural

86 information remains limited in $\mathrm{PA}^{11}$ and other type Tc toxins ${ }^{18,19}$. In clostridial binary

87 toxin, membrane prepore and pore structures have not been described. To understand

88 the protein translocation mechanism via the bacterial translocation channel, here we

89 determined the structures of the Ib heptameric translocation channel without and with

90 the enzymatic component Ia (Ib-pore and Ia-bound Ib-pore, respectively) by cryo-EM at

91 atomic resolution, providing the novel structural pose of Ia just prior to translocation in

92 the transmembrane spanning Ib-pore.

\section{Results}

\section{Ib oligomerization}

96 Ib oligomerization is rapidly induced at $37^{\circ} \mathrm{C}$ in Vero cells in a temperature but not $\mathrm{pH}$

97 dependent-manner ${ }^{20,21}$. Conversely, in vitro, $\mathrm{Ib}$ oligomerizes poorly at $37^{\circ} \mathrm{C}$ and/or

98 under acidic conditions following cleavage of the $20 \mathrm{kDa}$-terminal propeptide. PA

99 can oligomerize and form the PA-prepore upon propeptide cleavage, with the

100 conformational change from prepore to pore being induced at low $\mathrm{pH}$ in vitro ${ }^{22}$;

101 however, this leads to rapid and irreversible aggregation ${ }^{11,23}$. During cryo-EM sample

102 preparation in vitro, we found that $10 \%$ ethanol could efficiently induce oligomerization

103 upon N-terminal propeptide cleavage (Extended Data Fig. 1). Therefore, the sample was

104 prepared by adding $10 \%$ ethanol and $0.03 \%$ lauryl maltose neopentyl glycol (LMNG)

105 (Anatrace) following propeptide cleavage. Consequently, EM imaging showed that it

106 led to the direct conversion of monomeric Ib to the pore, not the prepore. The obtained 
107 Ib-pore was stable at neutral $\mathrm{pH}$, allowing cryo-EM structural analysis of the Ib-pore

108 along with the Ia-bound Ib-pore.

109

\section{Ib-pore and Ia-bound Ib-pore cryo-EM data}

111 Two data sets were collected using Titan Krios (FEI). With the first, we revealed the

112 Ib-pore structure using 38,433 particles after classification of 299,491 extracted

113 particles; local resolution analysis by Resmap ${ }^{24}$ showed the inner pore region at

114 approximately $2.5 \AA$ A resolution (Fig. 1a, Extended Table 1, Extended Data Fig. 2). As

115 described in Online Methods, the first data also included small amounts of Ia-bound

116 Ib-pore particles, which provided a $5.2 \AA$ resolution map of the Ia-bound Ib-pore

117 (Extended Data Fig. 2).

118 Therefore, we generated another data set, raising the ratio of Ia. Two classes clearly

119 showed Ia density on the Ib-pore with long $\beta$-barrel stem and short $\beta$-barrel stem,

120 respectively. Therefore, using 135,359 particles for short stem class and 62,940 particles

121 for long stem class following classification of 871,264 extracted Ia-bound Ib-pore

122 particles, 3D refinement was performed after several additional classifications

123 (Extended Data Fig. 3). Finally, 2.8 A map (Ia-bound Ib-pore with short stem) and 2.9

$124 \AA$ A map (Ia-bound Ib-pore with long stem) were yielded.

125

126 Structure of the Ib-pore

127 The obtained cryo-EM map shows the Ib-pore having a funnel-like structure, lacking

128 the lower stem region (328-365), suggesting that the bottom half of the stem had not yet

129 formed. Thus, the Ib-pore structure contains a stem of $40 \AA$ length ( $90 \AA$ at full length,

130 as noted for the Ia-bound Ib-pore), with the stem diameter the same as that of PA (>15 
131 A) (Fig. 2a-c; Extended Data Fig. 4a, 5a). The Ib-pore consists of four domains, 1'

132 (domain 1 without the propeptide), 2, 3, and 4, as with PA. The main pore body

133 comprises domain 2, which consists of two parts designated as 2c (residues 296-311

134 and 381-512) and 2s (residues 312-380) (Fig. 2a, c). Domain 2s is an extended $\beta$

135 -hairpin, seven copies of which assemble to form a membrane-spanning 14-stranded

$136 \beta$-barrel. Domain 3 is located at an intermediate position between domains $1^{\prime}$ and $2 \mathrm{c}$.

137 The cryo-EM density of domain 4, the receptor-binding domain, is weak and has the

138 lowest resolution among all four domains (Extended Data Fig. 2), likely owing to its

139 flexibility resulting from minimal contact with the other domains. The overall structure

140 of the Ib-pore is similar to that of the PA-pore (Extended Data Fig. 5a). Specifically, the

141 funnel structure consisting of domains $1^{\prime}, 2$, and 3 shows high similarity with that of the

142 PA-pore. Domains 1 and 2 of PA share 41 and $40 \%$ sequence identity with the

143 corresponding regions of Ib (Extended Data Fig. 6). The weak map density of domain 4

144 is also reported for the PA-pore ${ }^{11}$. In Ib, the relative position of this density differs from

145 that of PA-pore domain 4 (Extended Data Fig. 5a). As domain 4 is a receptor binding

146 domain, the sequence identity shows $<10 \%$ identity with PA, with the size also

147 differing (Ib domain 4 is twice as large as that of PA).

148 The cryo-EM density of the inner side of the funnel was analysed at high resolution;

149 thus, the side chain is clearly visible (Extended Data Fig. 4b). The narrowest clamp is

150 formed by seven F454s from seven protomers with a diameter of $6 \AA$, termed the

$151 \phi$-clamp in PA (Fig. 2e). Two additional bottlenecks exist in the cis-side: Ca-edge

152 (216-224), an N-terminal Ca binding site, and NSQ-loop (490-492), with $45 \AA$ and 23

$153 \AA$ diameters, respectively (Fig. 2d). Ca-edge, which is a unique di-calcium binding site 
154 (Extended Data Fig. 5b), and NSQ-loop are the most important regions for Ia-binding as

155 described later. We then divided the inner pore surface as layer I, II, III, IV, and V from

156 the cis- to trans-side (Fig. 2c). Layer I represents the broadest area of the funnel from

157 the Ca-edge to NSQ-loop. Layer II is from the NSQ-loop to E498-loop. Layer III is

158 from the E498-loop to $\phi$-clamp. Layer IV is from the $\phi$-clamp to H313 in the trans-side.

159 We designated the stem region as layer $\mathrm{V}$. The long stem with a $\beta$-barrel is created by

160 an amphipathic flexible loop (E312-I380) of the prepore.

161 The enzymatic component Ia (ADP-ribosyltransferase) is translocated from the cis

162 (layer I, II, and III) to the trans-side (layer IV and V). Although the sequence is not

163 well-conserved on the inner surface between the Ib-pore and PA-pore, structural

164 similarities exist and the negative $(\mathrm{pH} 7)$ and positive $(\mathrm{pH} 5.5)$ surface potential in the

165 cis side (Layer II and III) are maintained in both channels (Extended Data Fig. 5c). In

166 PA, N422 and D425 (422-NAQDDFSST-430) were noted as constituting an essential

$167 \mathrm{pH}$ sensor to lead the conversion from prepore to pore; in $\mathrm{Ib}$, these two residues are

168 conserved. Notably, H313 locates on the inner surface of the upper stem, which appears

169 to have an important function for translocation (Fig. 2c). Moreover, numerous Ser and

170 Thr residues exist in the inner surface stem region, which may have vital functions as

171 with PA although the residue positions are not conserved between Ib and PA (stem

172 region Ser/Thr content of 56\% each in $\mathrm{Ib}$ and $\mathrm{Pa}$ ) (Extended Data Fig. 5c).

\section{Structure of the Ia-bound Ib-pore}

175 Two separated classes, Ia-bound Ib-pore with short and long stem, were obtained from

176 the second data set by $\mathrm{C} 1$ data analysis (Extended Data Fig. 3). In both structures, Ia sits 
177 on the cis-side of the Ib-pore with the same binding mode (Fig. 1b-c and 3a-c; Extended

178 Data Fig. 4a). Notably, the Ia stoichiometry and binding mode differed from those of

179 the LF-bound PA-pore (PA:LF = 8:4 or 7:3). Clearly, one Ia binds to the Ib-pore

180 heptamer. Half of the Ia N-terminal domain is buried in the Ib-pore through several

181 interactions with interface $1650 \AA^{2}$. They primarily interact asymmetrically with

182 Ca-edges and NSQ-loops. Specifically, five Ca-edges in subunit C-G contribute to Ia

183 binding, with seven NSQ-loops in subunit A-G supporting the Ia N-terminal domain

184 (Fig. 3d-g; Extended Data Fig. 7).

185 No large Ia-induced structural change occurs in the Ib-pore between the apo-Ib-pore

186 and Ia-bound Ib-pore; only two NSQ-loops (E and F subunits) exhibit asymmetric

187 positional change (Fig. 3f). Moreover, no large structural change occurs in almost the

188 entire Ia molecule except the N-terminal (1-44) region between apo-Ia and the Ia-bound

189 Ib-pore (Fig. 3h). Within the seven NSQ-loop interaction, one (F subunit) pushes

190 residues 29-32 in the $\mathrm{N}$-terminal $\alpha$-helices (Ia), causing the apparent tilt and partial

191 unfolding of the N-terminal $\alpha$-helix (Fig. 3h; Extended Data Fig. 7). Thus, the

192 N-terminal region (1-17) in Ia unfolds in the Ib-pore because it is too large to fit in the

193 NSQ-loop bottleneck, then continues to the Ib-pore $\phi$-clamp gate (Fig. 3h, i). It appears

194 that the Ia molecule floats from the Ib protomers via NSQ-loops, thus providing free

195 inner space to accommodate the Ia N-terminal region. In summary, the main

196 interactions are caused by several asymmetrical Ca-edges and NSQ-loops. No specific

197 strong interaction exists between Ia and the Ib-pore, suggesting that this may constitute

198 an essential feature for the translocation channel and substrate protein, with the weak

199 interactions affording efficient translocation. 
200 Although detailed reports regarding Ia-Ib binding are lacking, an earlier study

201 showed that the N-terminal domain (residue 216-321 in domain $1^{\prime}$ ) of Ib is essential for

202 Ia docking ${ }^{25}$. This cryo-EM study of the Ia-bound Ib-pore provides precise information

203 regarding Ia and Ib-pore interactions, showing the Ca-edge of domain 1' along with the

204 NSQ-loop of domain 2 as essential for binding. It was also reported that Ib lacking just

205 the first N-terminal 27 residues did not facilitate Ia entry ${ }^{25}$. This is because the

206 N-terminal 27 residues form the Ib-pore $\mathrm{Ca}^{2+}$ binding site (Ca-edge) (Extended Data Fig.

207 5b). Moreover, residues 129-257 were proposed as the minimal Ia fragment for

208 translocation $^{26}$. The present Ia-bound Ib-structure showed that a native ordered structure

209 of the Ia N-terminal domain (45-210) is necessary for the stacking via NSQ-loops.

210 We next compared previous mutational results of $\mathrm{Ib}$ with the present $\mathrm{Ib}$-pore

211 structure. F454A led to loss of cytotoxicity and markedly increased single-channel

212 conductance, suggesting that the $\phi$-clamp is highly conserved and crucial for binary

213 toxin activity ${ }^{27}$. Several mutations within the amphipathic $\beta$-strand forming the stem

214 affected pore formation, single-channel conductance, and ion selectivity (S339E-S341E,

215 Q345H, and N346E ${ }^{27}$. Based on the structure, S339, S341, and N346 are located on the

216 inner stem surface, whereas Q345 is found on the stem tip. Ser and Thr residues on the

217 inner surface are likely essential for translocation.

219 Discussion

220 The available cryo-EM structure of $\mathrm{LF}_{\mathrm{N}}$-bound PA-pore $\left(\mathrm{PA}_{7}\left(\mathrm{LF}_{\mathrm{N}}\right)_{3}\right)$ is at low

221 resolution; nevertheless, the same binding mode as in the $\mathrm{LF}_{\mathrm{N}}$-bound PA-prepore is

222 assumed ${ }^{10,17}$. The first $\alpha$-helix and $\beta$-strand of $\mathrm{LF}_{\mathrm{N}}$ unfold and dock into the deep 
223 amphipathic cleft on the octamer surface, termed the ' $\alpha$-clamp' (Fig. 3j). Thus, the main

224 interactions are formed by the N-terminal helix binding to the PA-prepore $\alpha$-clamp.

225 Altough similar structural feature and electrostatic potential exist for Ib-pore

226 translocation channel, significant differences exist between Ia and LF binding. For LF,

227 the N-terminal $\alpha$-helix binds to the PA-pore $\alpha$-clamp, with the following $26 \mathrm{~N}$-terminal

228 residues being invisible owing to their flexibility. This flexibility is also observed in the

229 apo-structure, suggesting that the N-terminal region is intrinsically flexible (Fig. 3k).

230 Conversely, for Ia, binding to the channel causes Ia N-terminal unfolding. This indicates

231 that the Ib-pore serves as an unfolding chaperone for substrate translocation even at

232 neutral $\mathrm{pH}$. As the Ib $\alpha$-clamp site does not function as an $\alpha$-clamp, the Ib-pore rather

233 uses a novel mechanism for Ia N-terminal region unfolding: (1) a large portion of the

234 N-terminal domain of the Ia structure lies in the Ib-pore, and (2) the interaction induces

235 Ia N-terminal helix tilting and partial unfolding (Fig. 3h). Thus, the Ib-induced

236 disordered region (1-AFIERPEDFLKD-12) followed by unfolded N-terminal helix

237 (13-KENAI-17) directly continues to the $\phi$-clamp (Fig. 3i).

238 The Ia N-terminal region contains numerous hydrophobic along with both positive

239 and negative residues. Ia and LF share no sequence similarity, suggesting that the

240 characteristic residue assortment (positive, negative, and hydrophobic) is essential for

241 translocation. Constructs lacking both negative and positive charges in the unstructured

242 region of $\mathrm{LF}_{\mathrm{N}}$, composed of only Gly, Ser, and Thr, translocate more slowly and

243 independently of the $\Delta \mathrm{pH}$, indicating that a balance of acidic- and basic-charged

244 residues is required for efficient translocation with $\Delta \mathrm{pH}^{28}$. Furthermore, in endosomes,

245 as the phenylalanine clamp could be considered as a barrier of $\mathrm{pH}$ difference, the 
246 cis-side (Layer II and III) electrostatic potential is positive (pH 5.5), whereas the

247 trans-side (IV) electrostatic potential is negative (pH 7.0) (Extended Data Fig. 5c). The

248 electrostatic potential difference represents a common aspect in Ib-pore and PA-pore

249 and is likely important for translocation. It is also noted that electrostatic repulsion

250 between pore (NSQ-loop in subunit E) and substrate protein (Arg26) seems to be

251 important for efficient translocation (Extended Data Fig. 7b). Furthermore we consider

252 that the destabilization of Ca-edge at endosomal acidic $\mathrm{pH}$ is also key to reduce the

253 interaction, leading to more efficient translocation.

254 Despite the differences of binding stoichiometry and binding mode of $\mathrm{Ia} / \mathrm{Ib}$ and

255 LF/PA, the unfolded Ia and LF N-terminals are accommodated in electrostatically

256 charged cis-side pore, then led to the $\phi$-clamp. Therefore, we consider that the first entry

257 event, in which the tip of the unfolded N-terminus enters into the gate of the

258 hydrophobic $\phi$-clamp, is significant for translocation, similar to threading through a

259 needle. For Ia, the unfolded N-terminal region becomes freely accessible to the $\phi$-clamp

260 in the space of Layer III under the NSQ-loop. Thus, Ia N-terminal movement in the

261 limited space is beneficial compared with LF-bound PA with large open space, allowing

262 the hydrophobic tip to readily reach the hydrophobic $\phi$-clamp gate. This first event

263 appears necessary for an extended-chain Brownian ratchet model.

264 Two $\phi$-clamp configuration states (clamped and unclamped dilated states) have been

265 proposed that are allosterically regulated by the $\alpha$-clamp ${ }^{16,29}$. The allosteric

266 helix-compression model was proposed as more favourable than the extended-chain

267 Brownian ratchet model. This model explains that the successive $\alpha$-helix formation

268 induced by the $\alpha$-clamp is essential for substrate translocation and that newly formed 
$269 \alpha$-helices pass through the dilated $\phi$-clamp, leading to produce more power stroke.

270 However, structures of both the PA-pore at acidic $\mathrm{pH}$ and $\mathrm{Ib}$-pore at neutral $\mathrm{pH}$ show

271 that the $\phi$-clamp forms the same configuration as in the closed (clamped empty) state.

272 The dilated (unclamped empty) state structure has not yet known; moreover, whether

273 the dilated state exists and the allosteric helix-compression model is generalizable

274 remain controversial ${ }^{30,31}$. In Ib and C2II, the dilated state has not been observed by

275 electrophysiological study ${ }^{32,33}$ or in the present cryo-EM studies. With regard to its

276 structure, as seven phenylalanines are stacked in the $\phi$-clamp structure, change to a

277 more dilated conformation is unlikely. In addition to these, Ib does't use the $\alpha$-clamp for

$278 \alpha$-helices binding. Together, these observations indicate that the Ib translocation likely

279 occurs via a static $\phi$-clamp pore, suggesting the extended-chain translocation of the

280 unfolded N-terminal substrate (Fig. 4). The presented binary toxin complex structure

281 and the mechanism of unfolded $\mathrm{N}$-terminal substrate translocation should be conserved

282 in other $C$. difficile, $C$. spiroforme, and C. botulinum binary toxins. Notably, our study

283 provides structural clues to develop inhibitors of these binary toxins, especially CDT

284 from human opportunistic pathogen $C$. difficile ${ }^{34-36}$ or iota-like toxin (CPILE/BEC) in

285 human food poisoning outbreaks ${ }^{37-39}$.

286 At neutral $\mathrm{pH}$, only a minor conductance decrease was observed upon Ia addition to

287 the membrane cis-side ${ }^{32}$. However, at pH 5.6, cis-side conductance decreased to

$288 \quad 30-40 \%$ of the open configuration conductance ${ }^{32}$. Ia-mediated Ib blockage occurred

289 only at pH 5.6, suggesting the translocation occurs only at acidic $\mathrm{pH}$. In our study, we

290 revealed the Ia-bound Ib-pore structure at neutral $\mathrm{pH}$. The disordered Ia N-terminal 
291 region tip reaches the $\phi$-clamp (Fig. 3i); however, it may not be entirely blocked but still

292 fluctuate in the pore.

293 In summary, the present structure captured the Ia-bound Ib-pore just before

294 translocation: The Ia N-terminal region exhibited Ib-induced unfolding. In future studies,

295 using the stable Ib-pore, we expect to capture the translocation state of Ia in the Ib-pore

296 under acidic conditions. 


\section{References}

2991 Aktories, K. et al. Botulinum C2 toxin ADP-ribosylates actin. Nature 322, 300 390-392 (1986).

3012 Vandekerckhove, J., Schering, B., Barmann, M. \& Aktories, K. Clostridium

302 perfringens iota toxin ADP-ribosylates skeletal muscle actin in Arg-177. FEBS

$303 \quad$ Lett 225, 48-52 (1987).

3043 Blocker, D., Behlke, J., Aktories, K. \& Barth, H. Cellular uptake of the 305 Clostridium perfringens binary iota-toxin. Infect Immun 69, 2980-2987 (2001).

3064 Tsuge, H. et al. Crystal structure and site-directed mutagenesis of enzymatic 307 components from Clostridium perfringens iota-toxin. J Mol Biol 325, 471-483 $308 \quad$ (2003).

3095 Tsuge, $H$. et al. Structural basis of actin recognition and arginine 310 ADP-ribosylation by Clostridium perfringens iota-toxin. Proc Natl Acad Sci U S $311 \quad$ A 105, 7399-7404 (2008).

3126 Collier, R. J. \& Young, J. A. Anthrax toxin. Annu Rev Cell Dev Biol 19, 45-70 313 (2003).

3147 Miller, C. J., Elliott, J. L. \& Collier, R. J. Anthrax protective antigen: 315 prepore-to-pore conversion. Biochemistry 38, 10432-10441 (1999).

3168 Petosa, C., Collier, R. J., Klimpel, K. R., Leppla, S. H. \& Liddington, R. C. 317 Crystal structure of the anthrax toxin protective antigen. Nature 385, 833-838 318 (1997).

3199 Lacy, D. B., Wigelsworth, D. J., Melnyk, R. A., Harrison, S. C. \& Collier, R. J. Structure of heptameric protective antigen bound to an anthrax toxin receptor: a role for receptor in pH-dependent pore formation. Proc Natl Acad Sci U S A 101, 13147-13151 (2004).

10 Feld, G. K. et al. Structural basis for the unfolding of anthrax lethal factor by

32511 Jiang, J., Pentelute, B. L., Collier, R. J. \& Zhou, Z. H. Atomic structure of anthrax protective antigen pore elucidates toxin translocation. Nature 521, 545-549 (2015).

32812 Krantz, B. A. et al. A phenylalanine clamp catalyzes protein translocation through the anthrax toxin pore. Science 309, 777-781 (2005).

33013 Krantz, B. A., Finkelstein, A. \& Collier, R. J. Protein translocation through the anthrax toxin transmembrane pore is driven by a proton gradient. J Mol Biol 355, 968-979 (2006). 
33314 Sun, J., Lang, A. E., Aktories, K. \& Collier, R. J. Phenylalanine-427 of anthrax

334

335

336

337

338

339

340

341

342

343

344

345

346

347

348

349

350

351

$352 \quad 21$

353

354

355

356

357

358

359

360

361

$362 \quad 25$

363

$364 \quad 26$

365

366

$367 \quad 27$

368 protective antigen functions in both pore formation and protein translocation. Proc Natl Acad Sci U S A 105, 4346-4351 (2008).

15 Basilio, D., Juris, S. J., Collier, R. J. \& Finkelstein, A. Evidence for a proton-protein symport mechanism in the anthrax toxin channel. J Gen Physiol 133 (2009).

16 Das, D. \& Krantz, B. A. Peptide- and proton-driven allosteric clamps catalyze anthrax toxin translocation across membranes. Proc Natl Acad Sci U S A 113, 9611-9616 (2016).

17 Machen, A. J. et al. Asymmetric Cryo-EM Structure of Anthrax Toxin Protective Antigen Pore with Lethal Factor N-Terminal Domain. Toxins (Basel) 9 (2017).

18 Gatsogiannis, C. et al. A syringe-like injection mechanism in Photorhabdus luminescens toxins. Nature 495, 520-523 (2013).

19 Meusch, D. et al. Mechanism of Tc toxin action revealed in molecular detail. Nature 508, 61-65 (2014).

20 Nagahama, M., Nagayasu, K., Kobayashi, K. \& Sakurai, J. Binding component of Clostridium perfringens iota-toxin induces endocytosis in Vero cells. Infect Immun 70, 1909-1914 (2002).

1 Stiles, B. G., Hale, M. L., Marvaud, J. C. \& Popoff, M. R. Clostridium perfringens iota toxin: characterization of the cell-associated iota $\mathrm{b}$ complex. Biochem J 367, 801-808 (2002).

22 Koehler, T. M. \& Collier, R. J. Anthrax toxin protective antigen: low-pH-induced hydrophobicity and channel formation in liposomes. Mol Microbiol 5, 1501-1506 (1991).

23 Vernier, G. et al. Solubilization and characterization of the anthrax toxin pore in detergent micelles. Protein Sci 18, 1882-1895 (2009).

24 Kucukelbir, A., Sigworth, F. J. \& Tagare, H. D. Quantifying the local resolution of cryo-EM density maps. Nat Methods 11, 63-65 (2014).

25 Marvaud, J. C. et al. Clostridium perfringens iota-toxin: mapping of receptor binding and Ia docking domains on Ib. Infect Immun 69 (2001).

26 Marvaud, J. C. et al. Clostridium perfringens iota toxin. Mapping of the Ia domain involved in docking with Ib and cellular internalization. J Biol Chem 277 (2002).

27 Knapp, O. et al. Residues involved in the pore-forming activity of the Clostridium perfringens iota toxin. Cell Microbiol 17, 288-302 (2015). 
36928 Brown, M. J., Thoren, K. L. \& Krantz, B. A. Charge requirements for proton gradient-driven translocation of anthrax toxin. J Biol Chem 286, 23189-23199 (2011).

37229 Brown, M. J., Thoren, K. L. \& Krantz, B. A. Role of the alpha Clamp in the Protein Translocation Mechanism of Anthrax Toxin. J Mol Biol 427, 3340-3349 (2015).

$37530 \quad$ Yamini, G. \& Nestorovich, E. M. Relevance of the alternate conductance states of anthrax toxin channel. Proc Natl Acad Sci U S A 114, E2545-E2546 (2017). Krantz, B. A. Reply to Yamini and Nestorovich: Alternate clamped states of the anthrax toxin protective antigen channel. Proc Natl Acad Sci U S A 114, E2545-E2546 (2017).

32 Knapp, O., Benz, R., Gibert, M., Marvaud, J. C. \& Popoff, M. R. Interaction of Clostridium perfringens iota-toxin with lipid bilayer membranes. Demonstration of channel formation by the activated binding component Ib and channel block by the enzyme component Ia. J Biol Chem 277, 6143-6152 (2002).

33 Schmid, A., Benz, R., Just, I. \& Aktories, K. Interaction of Clostridium botulinum $\mathrm{C} 2$ toxin with lipid bilayer membranes. Formation of cation-selective channels and inhibition of channel function by chloroquine. J Biol Chem 269, 16706-16711 (1994). toxin) by strains of Clostridium difficile. FEMS Microbiol Lett 186, 307-312 (2000).

35 Warny, M. et al. Toxin production by an emerging strain of Clostridium difficile associated with outbreaks of severe disease in North America and Europe. Lancet 366, 1079-1084 (2005).

Barbut, F. et al. Clinical features of Clostridium difficile-associated diarrhoea due to binary toxin (actin-specific ADP-ribosyltransferase)-producing strains. $J$ Med Microbiol 54, 181-185 (2005).

40038 Monma, C. et al. Four foodborne disease outbreaks caused by a new type of 401 enterotoxin-producing Clostridium perfringens. J Clin Microbiol 53, 859-867 (2015). 
40339 Yonogi, S. et al. BEC, a novel enterotoxin of Clostridium perfringens found in human clinical isolates from acute gastroenteritis outbreaks. Infect Immun 82, 2390-2399 (2014).

\section{Acknowledgements}

408 This work was supported by JSPS KAKENHI Grant Numbers 18 K06170 and

409 17K15095. This work was also supported by the Basis for Supporting Innovative Drug

410 Discovery and Life Science Research (BINDS) from the AMED and the

411 "Nanotechnology Platform" of the Ministry of Education, Culture, Sports. We thank H.

412 Murata for the initial purification of Ib and JI. Kishikawa for help in cryo-EM analysis.

413 HT thanks M. Nagahama and M. Oda for helpful comments on the studies.

\section{Author contributions}

416 T.Yamada, T.Yoshida, A.K., and H.T. participated in research design and data analyses;

417 T.Yamada prepared the Ib-pore and Ia-bound Ib-pore for cryoEM; T.Yamada, A.K.,

418 K.M., and K.I. performed cryoEM data acquisition and image processing; T.Yoshida

419 performed the atomic model building, structure refinement and analyses; all author

420 contributed to writing the manuscript and H.T. supervise the project.

\section{Author information}

423 Reprints and permissions information is available at www.nature.com/reprints

424 Competing interests: The authors declare no competing interests.

425

426 Correspondence and requests for materials should be addressed to corresponding H.T.

427 (tsuge@cc.kyoto-su.ac.jp) 


\section{Methods}

429 Ib and Ia expression and purification. The iota toxin $b(\mathrm{Ib})$ gene was cloned into

430 pGEX4T-1 without a signal peptide, and Ib was overexpressed in Escherichia coli

431 Origami. The transformant was cultured in super broth medium containing ampicillin

$432(50 \mu \mathrm{g} / \mathrm{ml})$, tetracycline $(12.5 \mu \mathrm{g} / \mathrm{ml})$, and kanamycin $(15 \mu \mathrm{g} / \mathrm{ml})$ at $37^{\circ} \mathrm{C}$ until $\mathrm{OD}_{600}$ of

4330.6 , then isopropyl $\beta$-D-1-thiogalactopyranoside (final $1 \mathrm{mM}$ ) was added followed by

434 culturing at $23^{\circ} \mathrm{C}$ for $16 \mathrm{~h}$. The harvested cells were resuspended in lysis buffer

435 containing $20 \mathrm{mM}$ Tris $\mathrm{pH} 8.0,150 \mathrm{mM} \mathrm{NaCl}, 2 \mathrm{mM} \mathrm{CaCl}_{2}$, and $5 \mathrm{mM}$ dithiothreitol,

436 and disrupted by sonication. After centrifugation at $180,000 \times g$ for $40 \mathrm{~min}$, the

437 supernatant was loaded onto a Glutathione Sepharose 4B resin (GE Healthcare) column.

438 After washing the column with lysis buffer, the bound protein was eluted by a buffer

439 containing $20 \mathrm{mM}$ Tris $\mathrm{pH} 8.0,150 \mathrm{mM} \mathrm{NaCl}$, and $10 \mathrm{mM}$ reduced glutathione. The

440 eluted fractions were concentrated to $19 \mathrm{mg} / \mathrm{ml}$ and the buffer was exchanged to $20 \mathrm{mM}$

441 Tris $\mathrm{pH}$ 8.0, $50 \mathrm{mM} \mathrm{NaCl}, 2.5 \mathrm{mM} \mathrm{CaCl}_{2}$.

442 The iota toxin a (Ia) gene was cloned into pET-15b or pET-21b to produce Ia with

443 N-terminal or C-terminal His-tag, respectively. Then, Ia was overexpressed in E. coli

444 BL21 Star (DE3). The transformant was cultured in super broth medium containing

445 ampicillin $(50 \mu \mathrm{g} / \mathrm{ml})$ at $37^{\circ} \mathrm{C}$ until $\mathrm{OD}_{600}$ became 1.5 and then isopropyl

$446 \beta$-D-1-thiogalactopyranoside was added (final $0.5 \mathrm{mM}$ ) followed by culturing at $37^{\circ} \mathrm{C}$

447 for $16 \mathrm{~h}$. The harvested cells producing each Ia were individually resuspended in lysis

448 buffer containing $20 \mathrm{mM}$ Tris $\mathrm{pH} 8.0,300 \mathrm{mM} \mathrm{NaCl}$, and $20 \mathrm{mM}$ imidazole, and

449 disrupted by sonication. After centrifugation at $180,000 \times g$ for $40 \mathrm{~min}$, the supernatant

450 was loaded onto a Ni-NTA agarose column. After washing the column with lysis buffer, 
451 the bound protein was eluted by a buffer containing $20 \mathrm{mM}$ Tris $\mathrm{pH} 8.0,300 \mathrm{mM} \mathrm{NaCl}$,

452 and $500 \mathrm{mM}$ imidazole. The eluted fractions were concentrated and the buffer

453 exchanged to that containing $20 \mathrm{mM}$ Tris $\mathrm{pH} 8.0,2 \mathrm{mM} \mathrm{CaCl}_{2}$ to load onto an HiTrap Q

454 HP $5 \mathrm{ml}$ column (GE Healthcare). After anion exchange purification, Ia with C-terminal

455 His tag was concentrated to $2.63 \mathrm{mg} / \mathrm{ml}$, and the buffer was exchanged to $10 \mathrm{mM}$ Tris

$456 \mathrm{pH} 8.0$ and $100 \mathrm{mM} \mathrm{NaCl}$. Alternatively, Ia with N-terminal His-tag was incubated with

4570.001 (unit/ $\mu \mathrm{g}$ Ia) thrombin for $16 \mathrm{~h}$ at room temperature. Ia, in which the $\mathrm{N}$-terminal

458 His-tag was cleaved, was loaded onto a Ni-NTA agarose column, then the flow-through

459 and wash fractions were collected. Collected fractions were concentrated to $22 \mathrm{mg} / \mathrm{ml}$

460 and buffer was exchanged to $10 \mathrm{mM}$ Tris $\mathrm{pH} 8.0$ and $100 \mathrm{mM} \mathrm{NaCl}$ using an Amicon

461 filter.

462 Sample preparation for the first data set. Ib oligomerizes poorly at neutral and acidic

$463 \mathrm{pH}$ in vitro after cleavage of the pre-sequence with $\alpha$-chymotrypsin. Ib (30 mg) was

464 treated with $30 \mu \mathrm{g} \alpha$-chymotrypsin for $1 \mathrm{~h}$ at room temperature. This reaction was

465 terminated by adding phenylmethylsulphonyl fluoride (PMSF) (final $1 \mathrm{mM}$ ). Then, Ia

466 with C-terminal His-tag was added to the Ib solution with three-fold molar excess and

467 incubated $1 \mathrm{~h}$ at $37^{\circ} \mathrm{C}$. The solution was loaded onto a Ni-NTA agarose column. We

468 expected that the elution fraction includes Ia-bound Ib oligomer, but it was failed

469 because they didn't coexist in high concentration of imidazole. From oligomerization

470 screening, we found that ethanol induced oligomerization of Ib efficiently after cleavage

471 of pre-sequence incubating with $\alpha$-chymotrypsin (Extended Data Fig. 1a). Accordingly,

472 we changed to use the ethanol oligomerization to apply the flow-through fraction

473 including Ib. Ethanol (final 10\%) and LMNG (final 0.03\%) was added to $1 \mathrm{mg} \mathrm{Ib}$ in the 
474 flow-through fraction and incubated at $37^{\circ} \mathrm{C}$ for 1 hour. In order to separate $\mathrm{Ib}$ oligomer

475 from other small proteins, the solution was loaded onto $10.5 \mathrm{ml}$ of Glycerol gradient

476 bed (10-30\% Glycerol, $50 \mathrm{mM}$ HEPES pH 7.5, $100 \mathrm{mM} \mathrm{NaCl}, 1 \mathrm{mM} \mathrm{CaCl}, 0.03 \%$

477 LMNG). After ultracentrifuge at $230,139 \times g$ for 16 hours, fractions were collected by

$478250 \mu 1$ from bottom. Fractions showing high molecular mass by sodium dodecyl

479 sulphate-polyacrylamide gel electrophoresis (SDS-PAGE) were collected and buffer

480 was exchanged to $10 \mathrm{mM}$ HEPES $\mathrm{pH} 7.5,1 \mathrm{mM} \mathrm{CaCl}_{2}$, and $0.01 \% \mathrm{LMNG}$ and

481 concentrated to $1.9 \mathrm{mg} / \mathrm{ml}$. This sample included $\mathrm{Ib}$-pore and a small amount of Ia

482 (Extended Data Fig. 1b, c).

483 Sample preparation for the second data set. During sample preparation for the first

484 data set, binding between Ia and the Ib-pore occurred and the LMNG concentration was

485 too high for cryo-EM data collection. To solve these problems, Ib was purified with a

486 smaller concentration of LMNG and Ia was added following Ib-pore purification. Ib (21

$487 \mathrm{mg}$ ) was cleaved by $1 \mu \mathrm{g} \alpha$-chymotrypsin for $1 \mathrm{~h}$ at room temperature. This reaction

488 was terminated by adding PMSF (final $1 \mathrm{mM}$ ). The solution was then treated with $10 \%$

489 ethanol and $0.03 \% \mathrm{LMNG}$ for $1 \mathrm{~h}$ at $37^{\circ} \mathrm{C}$. To separate $\mathrm{Ib}$ oligomer from other small

490 proteins, the solution was loaded onto $10.5 \mathrm{ml}$ of Glycerol gradient bed (10-30\%

491 Glycerol, $50 \mathrm{mM}$ HEPES pH 7.5, $100 \mathrm{mM} \mathrm{NaCl}, 1 \mathrm{mM} \mathrm{CaCl} 2,0.003 \%$ LMNG). After

492 ultracentrifugation at $230,139 \times g$ for $16 \mathrm{~h}, 250 \mu \mathrm{l}$ fractions were collected from the

493 bottom. Fractions showing high molecular mass by SDS-PAGE were collected and the

494 buffer was exchanged to $10 \mathrm{mM}$ HEPES pH 7.5, $1 \mathrm{mM} \mathrm{CaCl}_{2}$, and $0.003 \% \mathrm{LMNG}$

495 Purified Ib oligomer was concentrated to $2.4 \mathrm{mg} / \mathrm{ml}$, then Ia without His-tag was added

496 with three-fold molar excess at the final step (Extended Data Fig. 1d, e). 
497 Cryo-EM imaging of the Ib-pore for the first data set. Sample vitrification was

498 performed using a semi-automated vitrification device (Vitrobot Mark IV, Fisher

499 Scientific, Eindhoven, The Netherlands). A $2.6 \mu$ l aliquot of sample solution at a

500 concentration of $0.38 \mathrm{mg} / \mathrm{ml}$ (1/5 dilution of the sample) was applied to

501 glow-discharged Quantifoil R1.2/1.3 in the Vitrobot at 100\% humidity. The grid was

502 then automatically blotted once from both sides with filter paper for $4.5 \mathrm{~s}$ blot time. The

503 grid was then plunged into liquid ethane with no delay time. Cryo-EM imaging was

504 performed using a Titan Krios (Fisher Scientific) operating at $300 \mathrm{kV}$ acceleration

505 voltage and equipped with a Cs corrector (CEOS, $\mathrm{GmbH})$ and a direct electron detector

506 Falcon 3 (counted mode) (Fisher Scientific) in automated data collection mode at a

507 calibrated magnification of $1.13 \AA$ /pixel (magnification $\times 59,000)$ and dose of $50 \mathrm{e} / \AA^{2}$

508 (or $0.46 \mathrm{e} / \AA^{2}$ per frame) with total $84.09 \mathrm{~s}$ exposure time. The data were automatically

509 collected using EPU software with a defocus range of -0.8 to $-2.5 \mu \mathrm{m}$ and were

510 fractionated 108 movie frames.

511 Image processing of the Ib-pore for the first data set. A total of 2,120 images were

512 collected in the first data set. The movie frames were subsequently aligned to correct for

513 beam-induced movement and drift using MOTIONCORR2 $2^{40}$, and contrast transfer

514 function (CTF) were estimated using CTFFIND4 $4^{41}$. A total of 299,491 particle images

515 were automatically picked using Gautomatch (http://www.mrc-lmb.cam.ac.uk/kzhang/)

516 and several rounds of 2D classification and 3D classification were performed using

517 RELION $-3.0^{42}$. The best among the 3D classes in which clearly showed 7-fold

518 rotational symmetry in Ib-pore were subjected to 3D refinement with C7 symmetry. The

519 3D-refined structure was further CTF refined using the per-particle defocus and 
520 Bayesian polishing, which improved the resolution to $2.9 \AA$ and a B-factor of $-46 \AA^{2}$

521 without the substrate Ia (Extended Data Fig. 2a-e).

522 Image processing of the Ia-bound Ib-pore for the first data set. While the 3D class

523 reconstruction proceeded in Ib-pore analysis, we identified another class that showed Ia

524 density on the Ib-pore (1,735 particles). The class was subjected to $3 \mathrm{D}$ refinement and

525 used as a template for a second 3D classification with 154,378 particles. Classes that

526 exhibited density on the $\mathrm{Ib}$ pore were selected for processing using 3D refinement.

527 Around the $\mathrm{Ib}$ pore membrane spanning stem (outside of the stem), some blurred

528 density was observed that appeared irregularly in each class; therefore, it was subtracted

529 to increase the efficiency of classification. Subtracted particles were subjected to a third

$5303 \mathrm{D}$ classification, and then classes that contained 15,890 particles with strong density on

531 the Ib pore were subjected to 3D refinement initially without and then with a solvent

532 mask. Finally, an Ia-bound Ib map was generated at 5.2 A $\square$ resolution (Extended Data

533 Fig. 2f-j). This result prompted us to collect a second data set using the sample while

534 raising the ratio of Ia.

535 Cryo-EM imaging of the Ia-bound Ib-pore for the second data set. A $2.6 \mu \mathrm{l}$ aliquot

536 of sample solution at a concentration of $0.48 \mathrm{mg} / \mathrm{ml}$ (1/5 dilution of the sample) was

537 applied to glow-discharged Quantifoil R1.2/1.3. Other procedures including sample

538 vitrification, blotting, freezing, and Cryo-EM imaging were the same as described for

539 the first data set.

540 Image processing of the Ia-bound Ib-pore for the second data set. Image processing

541 was performed as described for the first data set unless otherwise stated. A total of

542 2,151 images were collected in the second data set. The movie frames were

543 subsequently aligned to correct for beam-induced movement and drift using 
544 MOTIONCORR2 ${ }^{40}$, and CTF were estimated using $\mathrm{GCTF}^{41}$. A total of 871,264 particle

545 images were automatically picked using Gautomatch and several rounds of 2D

546 classification were performed using RELION-3.0 $0^{42}$. A total of 335,767 particles in the

547 best class were subjected to 3D refinement, per-particle CTF refinement and Bayesian

548 polishing. The polished particles were subjected to 3D classification using 3D

549 refinement structure as the reference and divided into eight classes. The two classes

550 clearly showed Ia density on the Ib-pore with long stem and short stem, respectively.

551 These two classes were subjected to 3D refinement, per-particle CTF refinement and

552 Bayesian polishing. The resulting 3D structure was further subjected to no-align 3D

553 classification using a mask covering Ia. The final 3D refinement and postprocessing of

554 two classes yielded maps with global resolution of $2.91 \AA$ and B factor of $-29 \AA^{2}$

555 (Ia-bound Ib-pore with long stem) and $2.80 \AA$ and B factor of $-10 \AA^{2}$ (Ia-bound Ib-pore

556 with short stem), according to 0.143 criterion of the FSC (Extended Data Fig. 3).

557 Model building and refinement. An Ib-pore model with short stem was built using the

558 first data set. An initial rigid-body fit of PA structure (PDB ID: 3J9C) was applied into

559 the cryo-EM density map using UCSF Chimera ${ }^{43}$. The Ib-pore model was then

560 manually built by iterative rounds of model modification in $\mathrm{COOT}^{44}$ and refinement

561 using PHENIX Real Space Refinement with secondary structure restraint ${ }^{45}$. Model

562 building of Ib Domain 4 was not carried out because of the low resolution and lack of

563 available crystal structure.

564 An Ia-bound Ib-pore model with short stem was built using the cryo-EM density

565 map with partial stem from the second data set. Following initial model building and

566 rigid-body fit using the Ib-pore model from the first data and the crystallographic 
567 structure of Ia (PDB ID: 1GIQ), they were manually modified and refined by iterative

568 rounds of COOT and PHENIX as for model building of the Ib-pore with short stem.

569 Furthermore, an Ia-bound Ib-pore model with long stem was built using the

570 cryo-EM density map with intact stem from the second data set. Following rigid-body

571 fit of the Ib-pore model with short stem, the intact long stem was manually built using

572 COOT and they were modified and refined by iterative rounds of COOT and PHENIX.

573 Although the density of Ia (C-terminal domain) was insufficient for de novo model

574 building, the cryo-EM density maps with short and long stem showed that the Ia and Ib

575 conformations of the two maps were the same. Therefore, rigid-body fitting of the Ia

576 model from the Ia-bound Ib-pore with long stem was finally carried out using UCSF

577 Chimera. Then, the final Ia-bound Ib-pore with long stem model was refined using

578 PHENIX Real Space Refinement with secondary structure restraint.

579 In the manuscript, we discussed Ia binding using the Ia-bound Ib-pore model with

580 short stem because the cryo-EM density of Ia with short stem was clearer than that with

581 long stem. The structures with long and short stem are the same except for stem length.

582 The difference of cryo-EM density of Ia was likely attributed to the difference of

583 particle number (short:135,359 and long: 62,940).

584 All figures were prepared using PyMOL (https://pymol.org/2/), UCSF Chimera, and

585 UCSF ChimeraX ${ }^{46}$.

586

587 Data availability

588 Cryo-EM maps and coordinates were deposited in the Electron Microscopy Data Bank

589 and Protein Data Bank with the accession codes EMDB-0721 and PDB 6KLX for the 
590 Ib-pore, EMDB-0713 and PDB 6KLO for the Ia-bound Ib-pore with short stem, and

591 EMDB-0720 and PDB 6KLW for the Ia-bound Ib-pore with long stem.

592

593 References

59440 Zheng, S. Q. et al. MotionCor2: anisotropic correction of beam-induced motion

595 for improved cryo-electron microscopy. Nat Methods 14, 331-332 (2017).

59641 Zhang, K. Gctf: Real-time CTF determination and correction. J Struct Biol 193, $597 \quad 1-12$ (2016).

59842 Zivanov, J. et al. New tools for automated high-resolution cryo-EM structure 599 determination in RELION-3. Elife 7, e42166 (2018).

60043 Pettersen, E. F. et al. UCSF Chimera--a visualization system for exploratory 601 research and analysis. J Comput Chem 25, 1605-1612 (2004).

60244 Emsley, P. \& Cowtan, K. Coot: model-building tools for molecular graphics. 603 Acta Crystallogr D Biol Crystallogr 60, 2126-2132 (2004).

60445 Afonine, P. V. et al. Real-space refinement in PHENIX for cryo-EM and $605 \quad$ crystallography. Acta Crystallogr D Struct Biol 74, 531-544 (2018).

60646 Goddard, T. D. et al. UCSF ChimeraX: Meeting modern challenges in 607 visualization and analysis. Protein Sci 27, 14-25 (2018).

608

609

610 
a

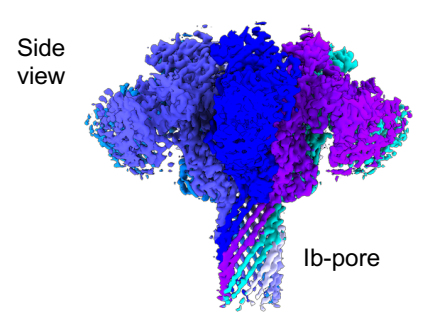

Top

view

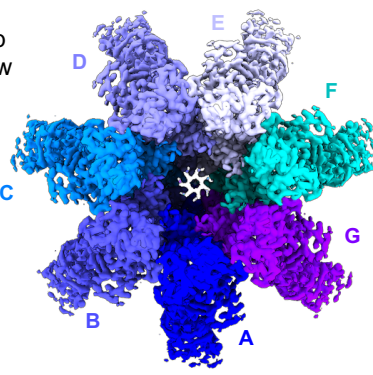

lb-pore
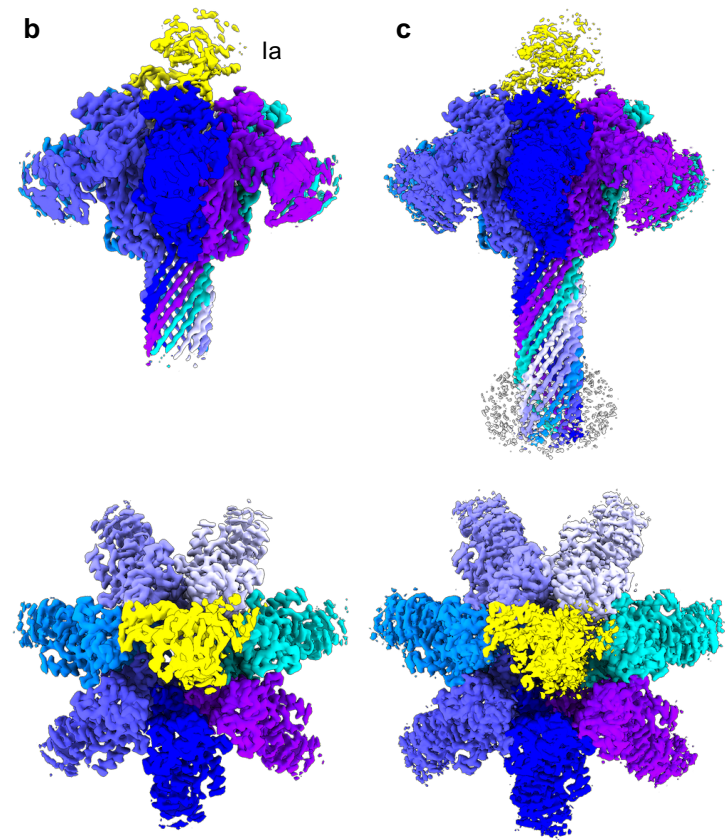

la-bound Ib-pore with short stem la-bound Ib-pore with long stem

Figure 1 | Cryo-EM density maps.

a, Ib-pore with short stem. b, Ia-bound Ib-pore with short stem. c, Ia-bound Ibpore with long stem. Ib protomers are shown in different colours. 

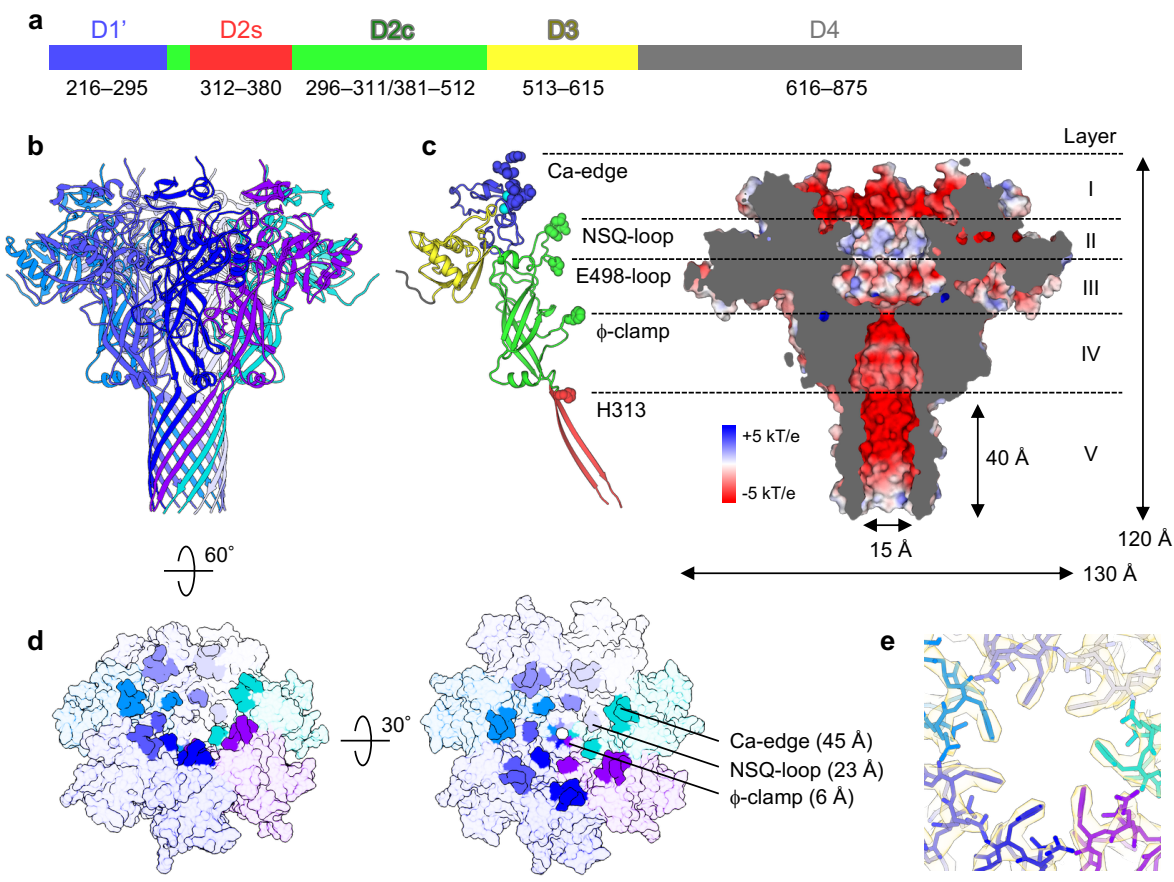

Figure 2 | Atomic model of the Ib-pore.

a, Ib Domain structure. b, Overall structure of the Ib-pore with short stem. The domain IV model was not built. c, Ib-pore layer structure. Five layers are shown in a protomer cartoon model colour-coded as in a along with the cut-away surface electrostatic potential at $\mathrm{pH}$ 7.0. The amino acid residues forming the boundary between adjacent layers are shown as a sphere model. d, Three bottlenecks of the Ib-pore. Ca-edge (216-224), NSQ-loop (490-492), and $\phi$ clamp (454). Surface model is shown. Diameters of bottlenecks are shown in parentheses. e, Top view of the f-clamp with cryo-EM density map. 
a
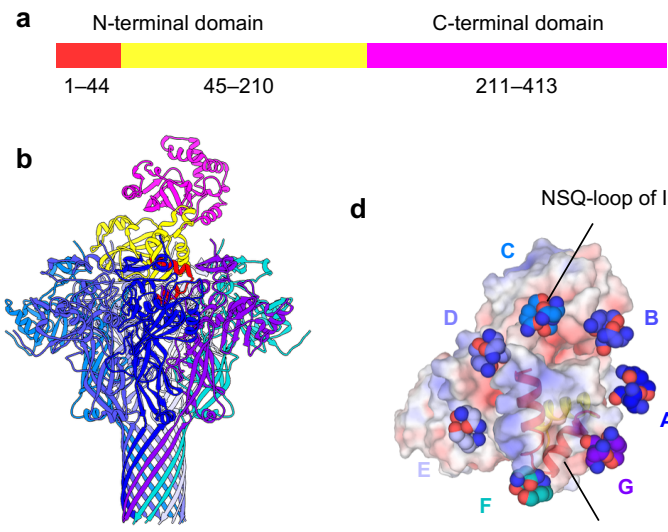

d

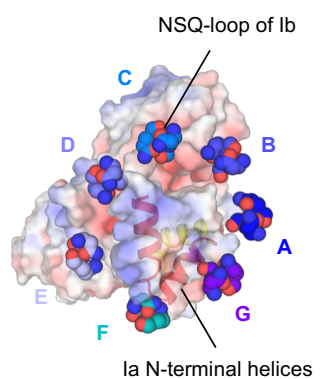

c

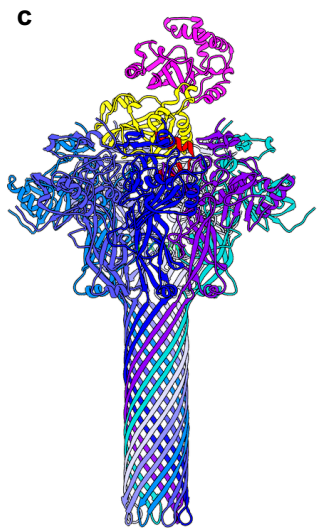

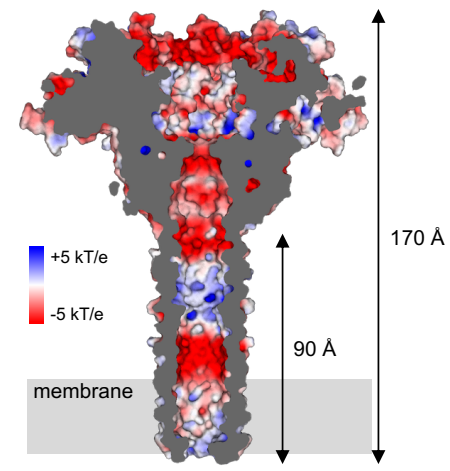
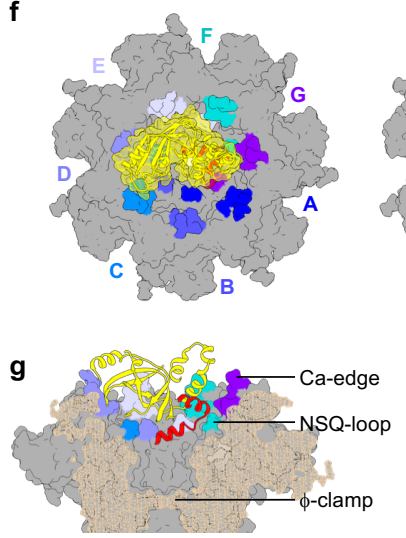

i

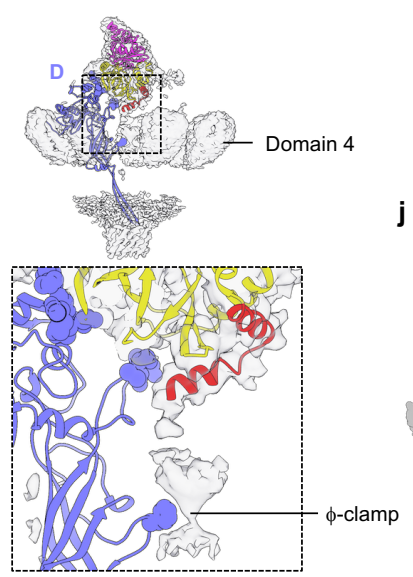

h
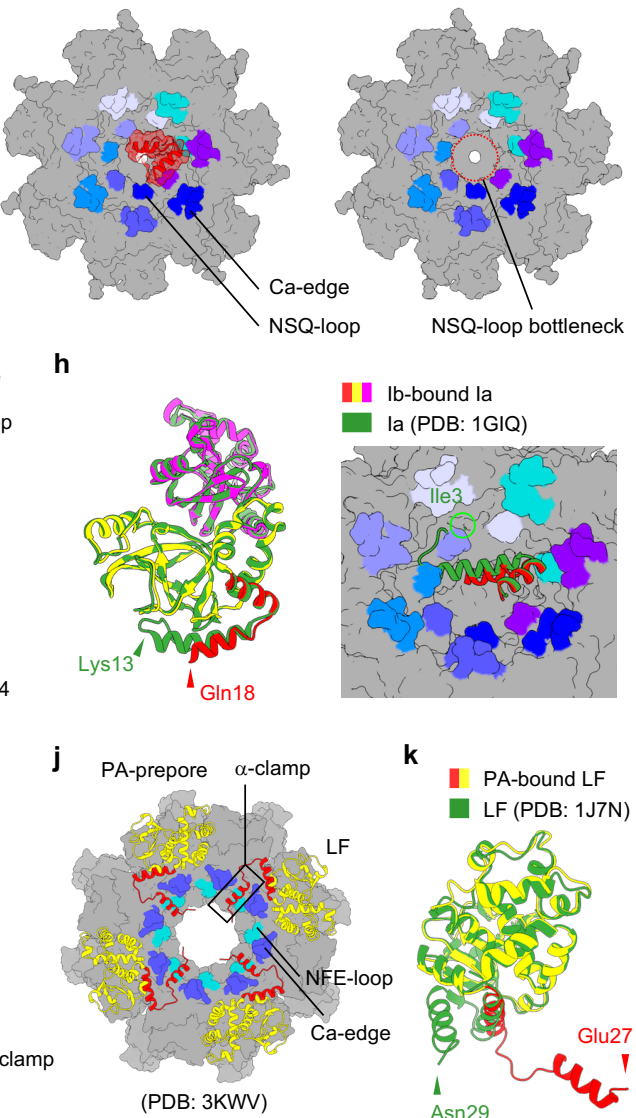

k LF (PDB: 1J7N)

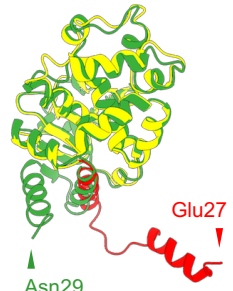
I PA-bound LF

Figure 3 | Atomic model of the Ia-bound Ib-pore.

a, Ia domain structure. b,c Ia-bound Ib-pore overall structures with short and long stems. d, Ia, bottom view. e, Cutaway surface electrostatic potential at $\mathrm{pH}$ 7.0. f, Top views. For clarity, 1-210 (left) and 1-44 (center) regions of Ia are shown. Ia is not shown (right). g, Cut-away view. h, Unfolding of Ia N-terminus. Ib-bound Ia and Ia crystal structure are superimposed. i, Extra map leading from the N-terminal helix to $\phi$-clamp. Map within $3 \AA$ of the Ib model is subtracted from the original map. j, LF-bound PA-prepore structure. k, PA-bound LF and LF crystal structure superposition. 
a

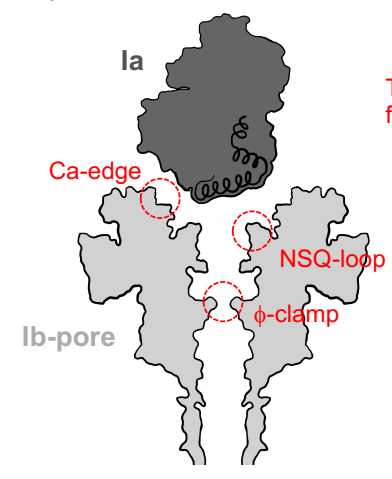

Step 1 b Complex formation

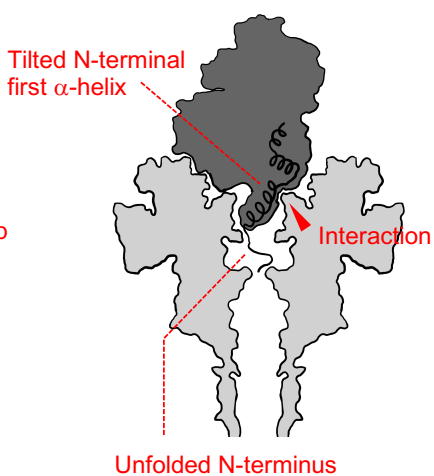

Step 2

Events at neutral $\mathrm{pH}$

c Initial translocation

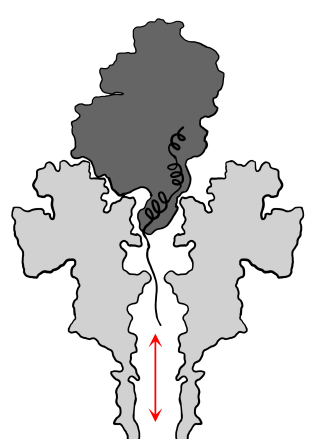

Extended-chain

Brownian ratchet model

Step 3 d Ongoing translocation

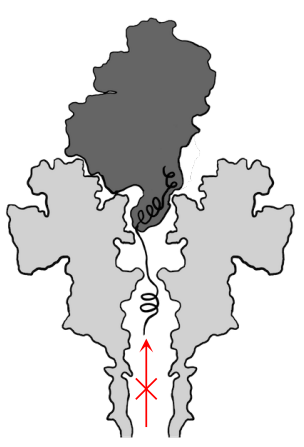

Step 4

Events at acidic $\mathrm{pH}$ in endosome

\section{Figure 4 | Translocation model of Ia via Ib-pore.}

Ia (black) and Ib-pore (grey). Arrows indicate possible Ia movement direction. a, Ia and Ib-pore before complexation. b, Ia-bound Ib-pore complex solved herein by single particle analysis. Ib-pore binding induces tilts and partial unfolding of the first N-terminal Ia $\alpha$-helix. c, Endosomal acidic conditions facilitate unfolded N-terminal tip entry into the Ibpore $\phi$-clamp followed by extended-chain Brownian ratchet model-mediated translocation. d, Expected mechanism to prevent Ia retro-translocation by $\alpha$-helix formation in the stem at neutral $\mathrm{pH}$ in the trans-side. 